\title{
The Influence of Magnetic Nanoparticles' Size on Trapping Efficiency in a Microfluidic Device
}

\author{
Alireza Bahadorimehr ${ }^{1}$, Zhila Rashemi ${ }^{2 *}$, Burhanuddin Yeop Majlis ${ }^{3}$ \\ ${ }^{1}$ Buein Zahra Technical University, Buein Zahra, Qazvin, Iran. \\ 2 Department of Electronics Engineering, Islamic Azad University, Arak Branch, Arak, Iran. \\ ${ }^{3}$ Institute of Microengineering and Nanoelectronics (IMEN), Universiti Kebangsaan Malaysia, 43600 UKM \\ Bangi, Selangor, Malaysia.
}

* Corresponding author. Tel.: +1-4168334928; email: zh.rashemi@yahoo.com

Manuscript submitted December 22, 2014; accepted February 27, 2015.

doi: 10.17706/ijbbb.2015.5.2.132-139

\begin{abstract}
Separation techniques in microfluidic devices using magnetic fields are well-known in biomedical applications in disease diagnosis like cancer, drug delivery, and hyperthermia. In this work, a microfluidic system is investigated. Here magnetic nanoparticles are separated from their medium using magnetic force. The whole microfluidic device for separation of functionalized magnetic beads is composed of a fluidic part and a magnetic part. The diameters of applied nanoparticles are $90 \mathrm{~nm}, 200 \mathrm{~nm}$ and $750 \mathrm{~nm}$. In this method, magnetic nanoparticles are injected into a microchannel, the permanent magnet is located under the channel and the magnetic field which is produced by permanent magnet is applied to the nanoparticles, so the magnetic nanoparticles are integrated above the permamnet magnet. Trajectory of particles, velocity, and the location in which magnetic nanoparticles are captured are obtained.
\end{abstract}

Key words: Magnetic nanoparticles, microfluidics, separation, trajectory.

\section{Introduction}

In recent years, microfluidic devices have been utilized in the field of biotechnology. Trapping, sorting, and manipulating of biological cells in a microfluidic channel is prodominant in lab-on-a-chip technology. For separation of fluid solutions into its components for biomedical applications such as DNA (Deoxyribonucleic acid) analysis and cell separations, using microfluididc devices is recommended. Although magnetism and microfluidics have been recently combined; but none of them is new. Many attentions have been received for Magnetic field-based bioseparation in microfluidic devices because of its great applications in biomedical research, clinical diagnostics and biotechnological sciences [1]-[4]. In the past few years, several magnetic particle-based microfluidic bioseparation systems have been developed for separation, analysis and detection of biomolecules [5], [6]. Since the magnetic susceptibility of native biological cells is not large or specific enough for separation, using magnetic beads has become one of the most common methods for magnetic cell separations. Magnetic nanoparticles have remarkable advantages. Their diameter can be controlled in the range of a few nanometers to tens of nanometers, thus they are able to be smaller than or comparable to a cell $(10-100 \mu \mathrm{m})$, a virus $(20-450 \mathrm{~nm})$, a protein $(5-50 \mathrm{~nm})$ or a gene (2 $\mathrm{nm}$ wide and 10-100 $\mathrm{nm}$ long). Therefore, magnetic nanoparticles can get closer or penetrate a biological entity. Permanent magnets or electromagnets are utilized for capturing of the functionalized particles in chosen points in in-vivo and in-vitro applications. 
There are many researches involved in using permanent magnets for magnetic particle capturing. A permanent magnet with $0.5 \mathrm{~T}$ remanent magnet, $5 \mathrm{~cm}$ length and $5 \mathrm{~mm}$ width has been used to capture 250 $\mathrm{nm}$ and $100 \mathrm{~nm}$ iron oxide nanoparticles at the distance of $1 \mathrm{~mm}$ away from the magnet (just below skin depth) [7]. The principle of the biosensor based on the DNA/RNA hybridization system and liposome signal amplification using permanent magnet is used in [8]. In most cases where a permanent magnet is used a famous sandwich principle is used for ELISA (Enzyme-Linked Immuno Assay) [9]-[13].

In this work, nanoparticles distribution prediction is focused on which is the most important priority in nanoparticles drug delivery modeling based on different literatures [14], [15]. But the evaluation of nanoparticles biodistribution is complicated because of several parameters including particle diameter, shape, surface chemistry, and local flow conditions. Size effect on distribution has been widely studied as an important parameter. For example, nanoparticles with diameter between 100 and $200 \mathrm{~nm}$ are ideal for tumor delivery due to the enhanced permeability and retention (EPR) effect [16], [17]. The influence of some important parameters such as inlet velocities of fluid containing magnetic nanoparticles, diameter of magnetic nanoparticles, magnetic field strength and the point of release of nanoparticles on the trapping efficiency are investigated [18]. In another work, the manipulation of magnetic beads in a microfluidic system that has one entrance and two channel for exit, is achieved by magnetic gradient fields resulting from conducting lines near the microfluidic channel for $0.5 \mu \mathrm{m}$ and $1.55 \mu \mathrm{m}$ particles [19]. Here, nanoparticles' trajectory is simulated. Trapping location and the influence of nanoparticles' diameter on trapping efficiency are investigated under the applied magnetic field using a permanent magnet. For this purpose, we simulate the movement of three different diameters of nanoparticles in the microchannel. Different parameters are studied which include: fluid behaviour, optimum values of magnetic field gradient, fluid velocity and height of microchannel. The microfluidic device has been fabricated by using well-known PDMS-SU8 method and tested by injection of nanoparticles.

\section{Design of Microfluidic Device}

In the magnetic separation, magnetic force is applied to the magnetic nanoparticles that move in the fluid, magnetic force absorbs nanoparticles and then it can be separated from fluid and trapped above the magnet in the microchannel. The design of microfluidic separation system which is used in this work is shown in Fig 1. It consists of two different parts, microfluidic and magnetic parts. The microfluidic part includes a microchannel with $100 \mu \mathrm{m}$ heights and $5 \mathrm{~mm}$ length, the fluid flow is assumed to be laminar in the microchannel. The magnetic part consists of a $1 \mathrm{~mm} \times 3 \mathrm{~mm}$ permanent magnet located under the microchannel to generate magnetic flux density around the microchannel. Relative permeability and magnetization of magnet are chosen, 1.05 and $480 \mathrm{KA} / \mathrm{m}$, respectively. In simulation, ten magnetic nanoparticles are injected into the microchannel, and their movement in presence of magnetic field is observed. In Fig. 1 magnetic flux density (Tesla) in all regions is shown by using the color bar.

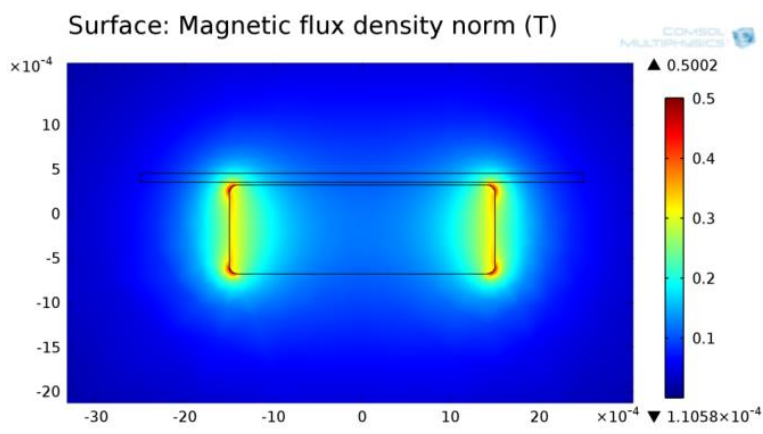

Fig. 1. Microfluidic system. 


\section{Equations}

\subsection{Magnetic Equations and Forces}

The most important parameters in nanoparticles magnetism are magnetic gradient and magnetic field. Since a magnet is used for separation of nanoparticles and according to the Lorants rule, a magnetic force is applied to the moving nanoparticles. The amount of magnetic field along the microchannel and in different distances is different, but in every part, there is a relation between magnetic field $(H)$ and magnetic flux density $(B)$ :

$$
H=\frac{B}{\mu_{0}}
$$

The applied magnetic force on nanoparticles along the microchannel depends on two factors, magnetic moment $m$ and magnetic flux density:

$$
\begin{aligned}
& F_{\text {mag }}=m \nabla B \\
& m=M s V
\end{aligned}
$$

where $M s$ is the magnetism of magnet and $V$ is the volume of a magnetic nanoparticle. According to (2), the magnetic force on nanoparticle can be increased by increasing the magnetic flux density.

The magnetic flux density curve along the microchannel, is shown in Fig. 2. As shown, the maximum amount of magnetic flux density occurs in the edges of the magnet and it is reduced in other parts.

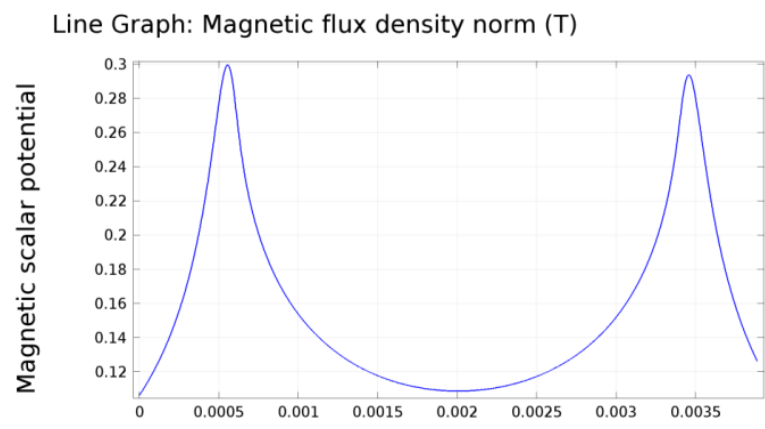

Fig. 2. Magnetic flux density in the microchannel.

\subsection{Fluid Equations and Forces}

The different forces are applied on nanoparticles in presence of magnetic flux density. One of them is magnetic force and others are the gravity force, Brownian force and the fluid force.

In most cases, the gravity force is ignored because of the small diameter of particles. In this work, it has not been considered too. For particles which are smaller than $90 \mathrm{~nm}$, the Brownian force is ignored. Therefore, two forces are applied on these magnetic nanoparticles. The fluid force is given by:

$$
F_{\text {Fluid }}=6 \pi r_{p} \eta\left(V_{\text {particle }}-V_{\text {Fluid }}\right)
$$

where, $r_{p}$ is the radius of particle, $V_{\text {particle }}$ and $V_{\text {Fluid }}$ are the velocity of particles and fluid, respectively. By equalizing (2) and (4), the minimum of magnetic field for trapping nanoparticles at constant flow rate can 
be obtained. Having $F_{\text {mag }}=F_{\text {Fluid, }}$ the final velocity can be calculated by:

$$
\Delta V=\frac{F_{\mathrm{mag}}}{6 \pi r_{p} \eta}=\frac{V \Delta \chi}{6 \pi r_{p} \eta \mu_{0}}
$$

This equation shows the relation between magnetic field and flow velocity, it is seen that doubling the diameter of the particles causes an increase in velocity by a factor of 4 (by substituting volume of sphere particles in this equation, the term $r_{p}{ }^{2}$ is appeared). The trajectory of nanoparticles movement can be obtained by (6). According to newton's second law:

$$
\begin{aligned}
& m \frac{d v}{d x}=F_{\text {mag }}+F_{\text {Fluid }} \\
& m=\rho_{\text {particle }} \frac{4}{3} \pi r_{p}^{3}
\end{aligned}
$$

where $m$ and $v$ are the mass of magnetic nanoparticle and the velocity of nanoparticle, respectively.

$$
m \frac{d v}{d x}=F_{\mathrm{mag}}-6 \pi r_{p} \eta \Delta v
$$

For the specific location of nanoparticle, the velocity of nanoparticles can be obtained by:

$$
v(t)=\frac{F_{\operatorname{mag}}}{6 \pi r_{p \eta}}\left(1-\exp \left(-\frac{6 \pi r_{p} \eta}{m}\right)\right)
$$

As shown in equations, the diameter of magnetic nanoparticles affects on absorption and velocity of nanoparticles.

According to (9), by increasing the diameter of nanoparticles, absorption of magnetic force can be increased.

In fluid dynamics, laminar flow occurs when a fluid flows in parallel layers. Reynolds number is an important factor in laminar flow. If fluid flows through a straight pipe, at a Reynolds number below a critical value, fluid motion will ultimately be laminar, whereas at larger Reynolds numbers, the flow can be turbulent. In this work, because of the low Reynolds number, fluid flow is considered laminar.

\section{Simulation of Motion and Nanoparticle Capturing in Microchannel}

The velocity of laminar fluid flow is considered to be $20 \mu \mathrm{m} / \mathrm{s}$. Permanent magnet is placed at $30 \mu \mathrm{m}$ under the microchannel. Ten nanoparticles are injected into the microchannel, the diameter of these nanoparticles is $90 \mathrm{~nm}$, and then simulation of the nanoparticles in presence of magnetic field is performed.

As shown in Fig. 3, only few nanoparticles in the microchannel are adsorbed to the surface of microchannel and are captured above the magnet while others continue to move along the microchannel. Here, each line shows the trajectory of the particles through the microchannel. The main purpose in this work is to show how the magnetic nanoparticles diameter affects the magnetic adsorption and separation in microfluididcs. To achieve this goal, we repeat this simulation with two different diameters of magnetic nanaoparticles, $200 \mathrm{~nm}$ and $750 \mathrm{~nm}$. 


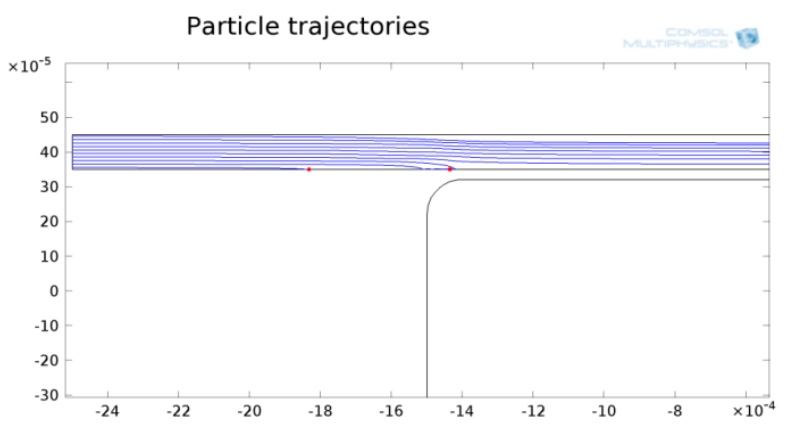

Fig. 3. Trajectory of $90 \mathrm{~nm}$ particles above the magnet.

Fig. 4 and Fig. 5 show the trajectory of $200 \mathrm{~nm}$ and $750 \mathrm{~nm}$ magnetic nanoparticles above the magnet, respectively. It is obvious that by increasing the nanoparticles diameter, the magnetic adsorption, capturing velocity of nanoparticles greatly increase.

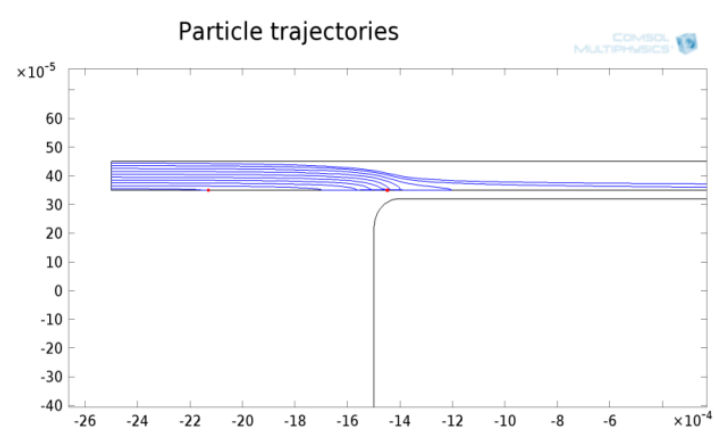

Fig. 4. Trajectory of $200 \mathrm{~nm}$ particles above the magnet.

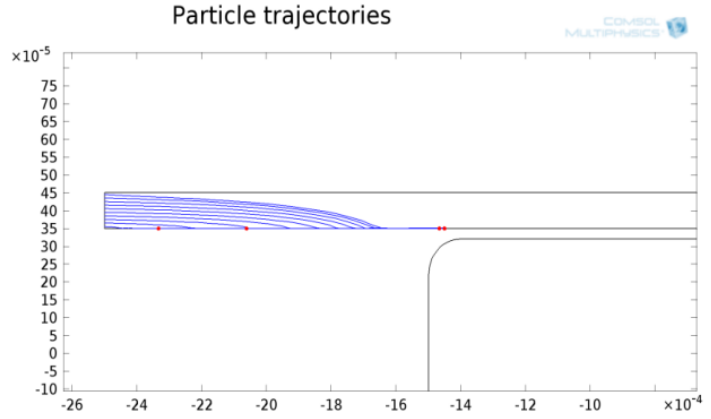

Fig. 5. Trajectory of 750nm particles above the magnet.

\section{Fabrication}

One of the most famous materials in microfluidic device fabrication is PDMS which can be patterned by using SU8 molding technique. This method has been used in most researches and considered as a primary method for fast, easy and reliable fabrication in microfluidic systems. SU8 was patterned on a silicon wafer with the thickness of $100 \mu \mathrm{m}$. Then PDMS is poured on this mold and can be released after some curing steps. We used corona discharge for PDMS-PDMS bonding. For magnetic part a neodymium magnet (NdFeB) was placed under the fluid channel for trapping purposes. Here, a $3 \mathrm{~mm} \times 3 \mathrm{~mm}$ of N52 neodymium magnet with $1 \mathrm{~mm}$ thickness has been chosen. This magnet is placed on a glass slide and covered by SU8. The thickness of this SU8 layer is equal to permanent magnet thickness. This layer was used to hold the magnet on glass and make a flat surface for fluidic part to place above it. The magnetic particles were injected to the microchannels inlets by using a programmable syringe pump (Longerpump TS-2A). The fluids are injected to the microfludic device via PTFE tubings.

The capturing process of $200 \mathrm{~nm}$ particles is shown in Fig. 6. As known from the magnetic part equations, these particles are the most difficult ones for capturing with minimum force exerted on them because of their sizes and magnetic susceptibilities. The side view of the particles' trajectory with our lab facilities was not applicable for this work. 

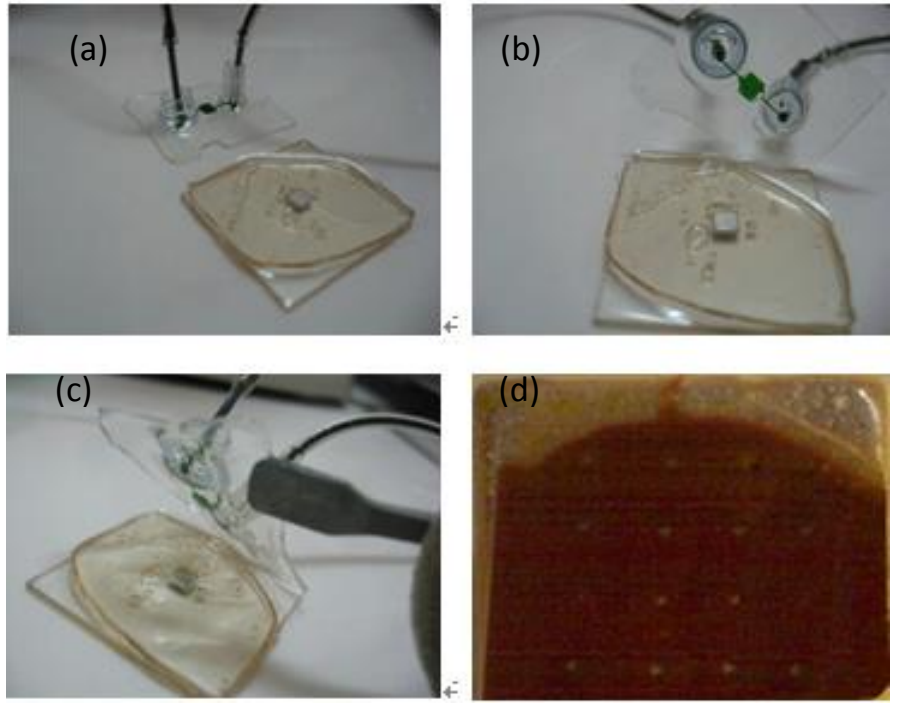

Fig. 6. (a), (b), (c) alignment of the fluidic and permanent magnet parts; (d) $200 \mathrm{~nm}$ particle capturing using permanent magnet.

\section{Results and Discussion}

This simulation was performed to observe the trajectory of magnetic nanoparticles with different diameters in microchannel in presence of magnetic field. Trajectory and trapping efficiency can be estimated by calculation of magnetic force and fluid force.

Different magnetic field patterns can be achieved by using multiple techniques in macro and micro scales. However, it is essential to differentiate between homogeneous and inhomogeneous magnetic fields. The density of flux lines is constant over a distance in a homogeneous magnetic field. Therefore, the gradient of the field is zero. However, in an inhomogeneous magnetic field, there are fluctuations in the density of magnetic flux lines over a distance. In this case, the gradient of magnetic field is not zero which is an advantage for magnetic particle capturing. This is because of the direct relation between the magnetic force on particles and the gradient of magnetic field which was shown in (2).

For $90 \mathrm{~nm}$ particles, the fluid and magnetic force were calculated and obtained, $0.02 \mathrm{pN}$ and $6.4 \mathrm{pN}$, respectively. The fluid force on nanoparticles increases by getting closer to the magnet and the laminar flow causes the nanoparticles not to move with the same velocity, so the particles near the walls move slower than other particles. As shown in the Fig. 2, the magnetic flux density exerted from the magnet is maximum in the edges of the magnet, which is due to the high density of magnetic dipoles in these areas, therefore, we expect that the most of the magnetic nanoparticles are trapped in the edges of the magnet. When the fluid force is equal to the magnetic force $(6.4 \mathrm{pN})$, nanoparticles on the surface of microchannel, above the magnet and in the inlet of the fluid, were trapped. We repeat this simulation for three different sizes of the nanoparticles. According to the equations, the magnetic force increases by increasing the volume of a magnetic nanoparticle (V). The maximum magnetic force on $90 \mathrm{~nm}, 200 \mathrm{~nm}$ and $750 \mathrm{~nm}$ particles were 6.4 $\mathrm{pN}, 70 \mathrm{pN}$ and $3.7 \mathrm{nN}$ above the edge of magnet, respectively. Trajectory of three sizes of nanoparticles is shown in Fig. 5, Fig. 6 and Fig. 7. They show that the trapping efficiency of larger particles $(750 \mathrm{~nm})$ was higher than the smellers' (200nm and $90 \mathrm{~nm}$ ) under similar condition including the applied magnetic field, inlet velocity and the height of microchannel. This is due to magnetic force and susceptibility of magnetic nanoparticles. The susceptibility of magnetic nanoparticles increases by increasing their sizes.

\section{Conclusion}


In this work, trajectory and capturing of magnetic nanoparticles in a microchannel of a microfluididc system above the magnet was predicted. The influence of nanoparticles' diameter on the magnetic adsorption and trapping efficiency were studied. This simulation and nanoparticles trajectory prediction is greatly beneficial for using in separation systems that are used in biomedical applications, especially in cancer treatment. In addition, it can help to choose the optimized diameter of nanoparticles to have more trapping efficiency for special applications. The fabricated device was also tested by using different particles and the capturing process was observed. Furthermore the time and velocity in which nanoparticles stop and capture in desire place in microchannel were estimated accurately.

\section{References}

[1] Pamme, N. (2007). Continuous flow separations in microfluidic devices. Lab on A Chip, 7(12), 1644-1659.

[2] Lee, H., Sun, E., Ham, D., \& Weissleder, R. (2008). Chip-NMR biosensor for detection and molecular analysis of cells. Nature Medicine, 14(8), 869-874.

[3] Smistrup, K., Hansen, O., Bruus, H., \& Hansen, M. F. (2005). Magnetic separation in microfluidic systems using microfabricated electromagnets-experiments and simulations. Journal of magnetism and magnetic materials, 293(1), 597-604.

[4] Alvankarian, J., Bahadorimehr, A., \& Majlis, B. Y. (2013). A pillar-based microfilter for isolation of white blood cells on elastomeric substrate. Biomicrofluidics, 7(1), 014102.

[5] Sivagnanam, V., Sayah, A., \& Gijs, M. A. (2008). Bead-based single protein micro-array realized through electrostatic self-assembly of carboxylated beads. Microelectronic Engineering, 85(5), 1355-1358.

[6] Gijs, M. A. M. (2004). Magnetic bead handling on-chip: new opportunities for analytical applications. Microfluidics and Nanofluidics, 1(1), 22-40.

[7] Nacev, A., Beni, C., Bruno, O., \& Shapiro, B. (2011). The behaviors of ferromagnetic nano-particles in and around blood vessels under applied magnetic fields. Journal of Magnetism and Magnetic Materials, 323(6), 651-668.

[8] Zaytseva, N. V., Goral, V. N., Montagna, R. A., \& Baeumner, A. J. (2005). Development of a microfluidic biosensor module for pathogen detection. Lab Chip, 5(8), 805-811.

[9] Herrmann, M., Veres, T., \& Tabrizian, M. (2006). Enzymatically-generated fluorescent detection in micro-channels with internal magnetic mixing for the development of parallel microfluidic ELISA. Lab Chip, 6(4), 555-560.

[10] Tang, D., Yuan, R., \& Chai, Y. (2007). Magnetic control of an electrochemical microfluidic device with an arrayed immunosensor for simultaneous multiple immunoassays. Clinical Chemistry, 53(7), 1323.

[11] Sista, R. S., Eckhardt, A. E., Srinivasan, V., Pollack, M. G., Palanki, S., \& Pamula, V. K. (2008). Heterogeneous immunoassays using magnetic beads on a digital microfluidic platform. Lab Chip, 8(12), 2188-2196.

[12] Chen, G. D., Alberts, C. J., Rodriguez, W., \& Toner, M. (2009). Concentration and purification of human immunodeficiency virus type 1 virions by microfluidic separation of superparamagnetic nanoparticles. Analytical Chemistry, 82(2), 723-728.

[13] Lincoln, B., Cordero, J. G., Poulsen, C., \& Lee, L. (2008). A microfluidic platform optimizing bead-based ELISA for the detection of cell secretion. Proceedings of the 12th International Conference on Miniaturized Systems for Chemistry and Life Sciences, San Diego, USA.

[14] Sanhai, W. R., Sakamoto, J. H., Canady, R., \& Ferrari, M. (2008). Seven challenges for nanomedicine. Nature Nanotechnology, 3(5), 242-244.

[15] Almeida, J. P. M., Chen, A. L., Foster, A., \& Drezek, R. (2011). In vivo biodistribution of nanoparticles. 
Nanomedicine, 6(5), 815-835.

[16] Cho, K., Wang, X., Nie, S., \& Shin, D. M. (2008). Therapeutic nanoparticles for drug delivery in cancer. Clinical Cancer Research, 14(5), 1310-1316.

[17] Mitragotri, S., \& Lahann, J. (2009). Physical approaches to biomaterial design. Nature Materials, 8(1), $15-23$

[18] Munir, A., Wang, J., \& Zhou, H. (2009). Dynamics of capturing process of multiple magnetic nanoparticles in a flow through microfluidic bioseparation system. Iet Nanobiotechnology, 3(3), 55-64.

[19] Weddemann, A., Wittbracht, F., Auge, A., \& Hütten, A. (2009). A hydrodynamic switch: Microfluidic separation system for magnetic beads. Applied Physics Letters, 94(17), 173501.

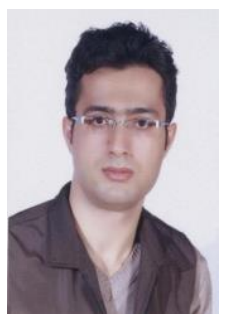

A. R. Bahadorimehr was born in Iran in 1982. He received his BSc and MSc in electronics engineering from Arak Azad University in 2004 and 2006, respectively. He received his $\mathrm{PhD}$ in nanoelectronics from UKM University in 2012 (Malaysia). Along with his industrial experience in different electrical and electronic engineering fields, he works on microfluidics, MRI and cancer therapy with some of his master students in electronics and biomedical engineering fields in different institutes and universities.

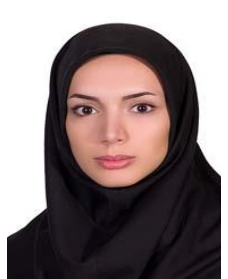

Z. Rashemi was born in Iran in 1989. She received her BSc and MSc in electronics engineering from Azad University in 2011 and 2014, respectively. Currently she investigates "Modeling of magnetic nanoparticles' movement in capillaries in presence of an external magnetic field". Her interests are fiber optics, cancer treatment issues, hyperthermia, and nanoparticle treatment methods.

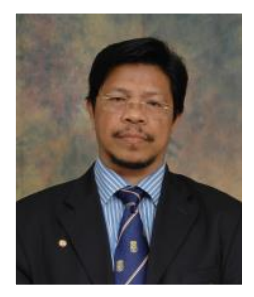

B. Y. Majlis is a professor of microelectronics at the Department of Electrical, Electronics and Systems Engineering. He received his Ph.D. in microelectronics from University of Durham, United Kingdom in 1988, the MSc in microelectronics from University of Wales, UK in 1980, and BSc (Hons.) in physics from UKM in 1979. He was a deputy dean of Engineering Faculty from 1995 until 1997. He is also a research fellow of Telekom Malaysia Research \& Development Division, and he was the director of UKM-TM Microelectronics Research Centre at the Faculty of Engineering, UKM. He was responsible in developing and planning the setting up of the clean room for research at UKM. He had attended intensive industrial training in GaAs MMIC design and manufacture at GEC-Marconi Material Technology Ltd. United Kingdom. He is a senior member of the Institution of Electrical and Electronics Engineer (IEEE) and the chairman of IEEE Electron Devices Malaysia Chapter from 1994 to 2006. He is also a fellow member of Malaysian Solid State Science and Technology (MSSS). He is the founder president of Malaysia Nanotechnology Association (MNA). He initiated research in microfabrication and microsensors at UKM in 1995 and has also initiated research in GaAs technology with Telekom Malaysia. In 2001 he initiated research in MEMS with substantial research funding of US\$10 million from Ministry of Science, Technology and Innovation. His current interests are design and fabrication of MEMS sensor, RFMEMS, BiOMEMS. Lab on Chip and microenergy and now is the program leader for MEMS research, a National Strategic Research Program. Now he is the director of Institute of Microengineering and Nanoelectronics (IMEN). 